\title{
Synthesis of POSS Derived Organic-Inorganic Hybrid Esters for Insulating Oil Applications
}

\author{
Kyeong-min Choi, S. J. Harshavardhan, Ch. Sridhar, B. V. D. Vijaykumar, \\ Deepak Kumar, Kiwan Jang, Man-Sig Lee, ${ }^{\dagger}$ and Dong-Soo Shin ${ }^{*}$ \\ Departments of Chemistry and Physics, Changwon National University, Changwon 641-773, Korea \\ *E-mail:dsshin@changwon.ac.kr \\ 'Green Technology Center, Korea Institute of Industrial Technology (KITECH), Ulsan 681-310, Korea \\ Received April 18, 2014, Accepted May 27, 2014
}

\begin{abstract}
In this work, a new family of polyhedral oligomeric silsesquioxanes (POSS) based esters have been synthesized that consists eight ester functional groups. These are classified into Type-I and Type-II esters based on the starting materials, octakis(3-chloropropyl)silsesquioxane (Cl-POSS) and octakis(3-hydroxypropyldimethylsiloxy) octasilsesquioxane (OHPS) respectively. These new POSS type ester moieties can be used as insulating oils and oil additives in the transformers and also considered to be alternatives to mineral and vegetable oils that are presently used in the insulation system.
\end{abstract}

Key Words : POSS esters, Esterification, Insulating oils

\section{Introduction}

A majority of power transformers that work at all ranges of voltage and power are oil power transformers, also called as dry transformers, which transfers energy from one circuit to another without any direct electrical connection. ${ }^{1}$ Insulation plays a major role in condition assessment and environmental risks connected with the transformer in service, where mineral oil is the most important component to be taken into consideration. Mineral oil is suitable for risk of electric shock, noise and vibration as well as energy and power losses. However, the increasing effects of these oils on environment seem to be hazardous day by day because of their poor degradability and high carcinogenic properties. ${ }^{2}$ In addition, they contaminate soil as well as sea waters when spilled out from the transformer and pose great difficulties in recovery and degradation. Mineral oils are estimated to face serious shortages by the mid-twenty-first century. Therefore, efforts towards the research to develop fully biodegradable insulating fluids were started in mid 1990s.

Natural esters and synthetic esters are considered the most likely candidate for a fully biodegradable insulating fluid. Natural esters, are the vegetable oils obtained from natural resources like canola, soya bean, corn, peanut, sunflower and olive, which are categorized as saturated and single, double and triple unsaturated fatty acid. ${ }^{3-5}$ Though, natural esters have many advantages over mineral oils which include higher biodegradability, high flash point etc., triple unsaturated fatty acids have lower viscosities and very unstable in oxidation. Synthetic esters are the oils from fatty acids and polyols that are environmentally benign alternatives for mineral oil based lubricants. These organic esters represent the largest single segment of the synthetic lube market which are designed for specific applications that include maximize stability, lower volatility, maintain low pour points and provide good additive compatibility. The carbon acid esters such as pentaerythrite tetra fatty acid ester 1 (Figure 1) are used in transformers, which were synthesized from polyols by using catalysts such as sulfuric acid, $p$-toluenesulfonic acid, Sn, Zr, Ti, alcolates, carboxylates and chelates. ${ }^{6}$ These processes need continuous monitoring and improvement in the quality of oils, because some of them cause significant acidity and also give dark colored products. Thus, a simple method for the efficient synthesis of ester based oils has always been in demand. Thus, synthetic esters gained much importance and are also promising to attain specific fluid properties that come from application oriented design.

On the other hand, polyhedral oligomeric silsesquioxane (POSS) skeletons are biodegradable nanostructures with an empirical formula $\mathrm{RSiO}_{1.5}$, which consists of a rigid and cubic silica core with $0.53 \mathrm{~nm}$ side length surrounded by eight organic corner groups, where $\mathrm{R}$ may be a hydrogen atom or an alkyl, alkylene, acrylate, epoxide or hydroxyl group. ${ }^{7,8}$ The use of POSS in polymer based nano compo-

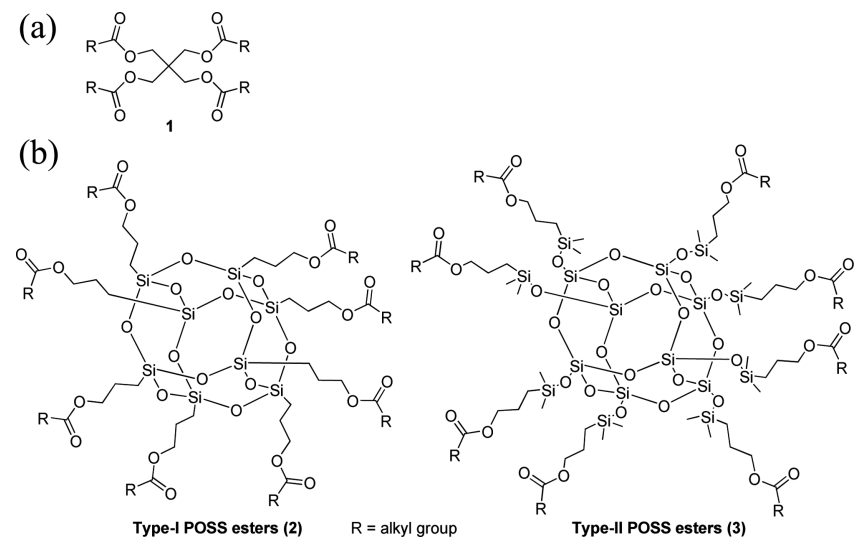

Figure 1. The chemical structures of (a) pentaerythritol ester and (b) POSS esters. 
sites is a promising issue for the design of materials with high-performance in several applications such as low-dielectric applications, ${ }^{9}$ flame resistant, ${ }^{10,11}$ oxidation resistant, ${ }^{12}$ surface hardening, ${ }^{13}$ photoluminescent, ${ }^{14}$ improved thermal and mechanical stability ${ }^{15-17}$ and also medical uses. ${ }^{18-22}$ Functionalized POSS play an important role in building molecular nano-composites. Our interest on the biodegradable lubricants encouraged us towards the synthesis of various biodegradable insulating oils from POSS based materials due to their properties mentioned above. Aliphatic ester moieties when attached to POSS expected to show good lubricant properties. Thus, we here in report the design and synthesis of new type of POSS based inorganic/organic hybrid insulating oils $\mathbf{2}$ and $\mathbf{3}$ (Figure 1) from POSS based chlorides and alcohols using two different and convenient reaction conditions.

\section{Experimental}

Materials and Methods. Butyric acid, 4-pentenoic acid, heptanoic acid, octanoic acid, 2-methylheptanoic acid and palmitic acid were purchased from Sigma Aldrich chemicals and used as received. Pentanoyl chloride, hexanoyl chloride, octanoyl chloride, cyclohexane carbonyl chloride were used after distillation, other reagents (potassium hydroxide, triethylamine, $\mathrm{N}, \mathrm{N}$-dimethylformamide, dichloromethane, tetrahydrofuran) were commercially available. Octakis(3-chloropropyl)silsesquioxane (Cl-POSS) and octakis(3-hydroxypropyldimethylsiloxy)octasilsesquioxane (OHPS) were synthesized in laboratory by known procedures. ${ }^{23-26}$

Instrumentation. Thermo gravimetric and thermal differential analyses (TG-DTA) of POSS oil samples were performed by a simultaneous SDT Q200 (USA) analyzer at a heating rate of $10{ }^{\circ} \mathrm{C} \mathrm{min}^{-1}$ under nitrogen gas flow. ${ }^{1} \mathrm{H}$ $\mathrm{NMR}$ and ${ }^{13} \mathrm{C}-\mathrm{NMR}$ spectra were recorded on a Bruker Avance-400 spectrometer.

Octakis(3-chloropropyl)silsesquioxane (5): Cl-POSS was synthesized by following published procedures. ${ }^{23,24} \mathrm{~A}$ solution of 3-chloropropyltrimethoxysilane (CLS) (39.7 g, $0.2 \mathrm{~mol}$ ) was added to a solution of dry methanol. To this mixture was added concentrated $\mathrm{HCl}(45 \mathrm{~mL})$, and the reaction mixture was kept at room temperature for 2 days. During which a catalytic amount of $\mathrm{PtCl}_{4}$ was added to this solution and was heated to $50{ }^{\circ} \mathrm{C}$. A crystalline precipitate formed after a day, which was washed with deionized water to afford $13.1 \mathrm{~g}$ of Cl-POSS in $40 \%$ yield. mp $207{ }^{\circ} \mathrm{C} .{ }^{1} \mathrm{H}$ $\mathrm{NMR}\left(\mathrm{CDCl}_{3}, 400 \mathrm{MHz}\right) \delta 3.85-3.42(\mathrm{~m}, 16 \mathrm{H}), 2.19-1.72$ $(\mathrm{m}, 16 \mathrm{H}), 1.01-0.67(\mathrm{~m}, 16 \mathrm{H}) .{ }^{29} \mathrm{Si} \mathrm{NMR}\left(\mathrm{CDCl}_{3}, 79.4 \mathrm{MHz}\right)$ $\delta-67.1 .{ }^{13} \mathrm{C}-\mathrm{NMR}\left(\mathrm{CDCl}_{3}, 100 \mathrm{MHz}\right) \delta 9.08,26.01,46.87$; IR(KBr): $v_{\max } 2955,2940,2876,1410,1273,1115,536,478$ $\mathrm{cm}^{-1}$.

General Procedure for Type-I POSS-Esters. To a solution of alkylcarboxylic acid $(2.31 \mathrm{mmol})$, in tetrahydrofuran $(10 \mathrm{~mL})$ was added $\mathrm{KOH}(2.31 \mathrm{mmol})$ at room temperature. The reaction mixture was stirred at room temperature to completion of the reaction $(\sim 0.5 \mathrm{~h})$ as indicated by TLC, then the solvent was evaporated under reduced pressure gave white solid. To this was added dry DMF ( $5 \mathrm{~mL})$, ClPOSS (300 mg, $0.28 \mathrm{mmol})$ and cat KI (5 mol \%) at RT. The reaction temperature was allowed to warm at $100{ }^{\circ} \mathrm{C}$ and continued until $\underline{\mathrm{CH}}_{2}-\mathrm{Cl}$ peak disappeared totally in the ${ }^{1} \mathrm{H}$ NMR spectra of samples taken from the reaction. Then temperature was slowly brought to room temperature, diethyl ether $(10 \mathrm{~mL})$ was added, washed with water $(2 \times 10$ $\mathrm{mL}$ ), dried over $\mathrm{Na}_{2} \mathrm{SO}_{4}$, filtered and concentrated gave the pure products.

Octakis(3-(butanoyloxy)propyl)octasilsesquioxane (2A): ${ }^{1} \mathrm{H}$ NMR (400 MHz, $\mathrm{CDCl}_{3}$ ) $\delta$ 4.06-4.0 (m, $\left.16 \mathrm{H}\right), 2.32-2.26$ $(\mathrm{m}, 16 \mathrm{H}), 1.76-1.56(\mathrm{~m}, 32 \mathrm{H}), 0.95(\mathrm{t}, J=7.32 \mathrm{~Hz}, 24 \mathrm{H})$, 0.70-0.59 (m, 16H); ${ }^{13} \mathrm{C} \mathrm{NMR}\left(\mathrm{CDCl}_{3}, 100 \mathrm{MHz}\right) \delta 173.5$, $68.83,36.14,22.15,18.56,13.65,8.70 ;{ }^{29} \mathrm{Si}$ NMR $(79.5$ $\left.\mathrm{MHz}, \mathrm{CDCl}_{3}\right) \delta-69.3$. FTIR (neat): $v_{\max } 2666,1736,1458$, $1260,1171,1127,762 \mathrm{~cm}^{-1}$.

Octakis(3-(pentenoyloxy)propyl)octasilsesquioxane (2B): ${ }^{1} \mathrm{H}$ NMR (400 MHz, $\mathrm{CDCl}_{3}$ ) $\delta$ 5.90-5.75 (m, $\left.8 \mathrm{H}\right), 5.10-4.96$ $(\mathrm{m}, 16 \mathrm{H}), 4.11-3.98$ (m, $16 \mathrm{H}), 2.48-2.30$ (m, $32 \mathrm{H}), 1.80-$ $1.56(\mathrm{~m}, 16 \mathrm{H}), 0.74-0.55(\mathrm{~m}, 16 \mathrm{H}) ;{ }^{13} \mathrm{C} \mathrm{NMR}\left(\mathrm{CDCl}_{3}, 100\right.$ $\mathrm{MHz}) \delta 172.81,136.66,115.46,66.06,33.62,28.85,22.27$, 8.08; ${ }^{29} \mathrm{Si} \mathrm{NMR}\left(79.5 \mathrm{MHz}, \mathrm{CDCl}_{3}\right) \delta-69.1$. FTIR (neat): $v_{\max } 3082,2925,1736,1643,1446,1272,1127,750 \mathrm{~cm}^{-1}$.

Octakis(3-(heptenoyloxy)propyl)octasilsesquioxane (2C): ${ }^{1} \mathrm{H}$ NMR (400 MHz, $\left.\mathrm{CDCl}_{3}\right) \delta$ 4.06-.98 (m, $\left.16 \mathrm{H}\right), 2.33-2.27$ $(\mathrm{m}, 16 \mathrm{H}), 1.76-1.55(\mathrm{~m}, 32 \mathrm{H}), 1.36-1.26$ (m, $48 \mathrm{H}), 0.92-$ $0.84(\mathrm{~m}, 24 \mathrm{H}), 0.70-0.50(\mathrm{~m}, 16 \mathrm{H}) ;{ }^{13} \mathrm{C} \mathrm{NMR}\left(\mathrm{CDCl}_{3}, 75\right.$ MHz) $\delta 173.67,65.94,34.34,31.53,28.89,24.98,22.54$, $22.40,13.98,8.67 ;{ }^{29} \mathrm{Si}$ NMR $\left(79.5 \mathrm{MHz}, \mathrm{CDCl}_{3}\right) \delta-69.3$. FTIR (neat): $v_{\max } 2925,1730,1463,1272,1196,1121,750$ $\mathrm{cm}^{-1}$.

Octakis(3-(octanoyloxy)propyl)octasilsesquioxane (2D): ${ }^{1} \mathrm{H}$ NMR (400 MHz, $\left.\mathrm{CDCl}_{3}\right) \delta$ 4.09-3.97 (m, $\left.16 \mathrm{H}\right), 2.30-2$ $(\mathrm{t}, J=7.32 \mathrm{~Hz}, 16 \mathrm{H}), 1.67-1.56(\mathrm{~m}, 32 \mathrm{H}), 1.37-1.19(\mathrm{~m}, 64$ $\mathrm{H}), 0.88(\mathrm{t}, J=7.32 \mathrm{~Hz}, 24 \mathrm{H}), 0.72-0.59(\mathrm{~m}, 16 \mathrm{H}) ;{ }^{13} \mathrm{C}$ $\mathrm{NMR}\left(\mathrm{CDCl}_{3}, 100 \mathrm{MHz}\right) \delta 173.70,65.94,34.31,31.72$, $29.22,28.98,25.03,22.62,22.40,14.05,8.65 ;{ }^{29} \mathrm{Si} \mathrm{NMR}$ $\left(79.5 \mathrm{MHz} \mathrm{CDCl}_{3}\right) \delta-69.3$. FTIR (neat): $v_{\max } 2925,1736$, $1469,1272,1191,1116,750 \mathrm{~cm}^{-1}$.

Octakis(3-(2-methylheptanoyloxy)propyl)octasilsesquioxane (2E): ${ }^{1} \mathrm{H}$ NMR $\left(400 \mathrm{MHz}, \mathrm{CDCl}_{3}\right) \delta$ 4.09-4.0 (m, 16 $\mathrm{H}), 2.47-2.38(\mathrm{~m}, 8 \mathrm{H}), 1.77-1.61(\mathrm{~m}, 24 \mathrm{H}), 1.48-1.25(\mathrm{~m}$, $56 \mathrm{H}), 1.22-1.14$ (m, $24 \mathrm{H}), 0.93-0.87$ (m, $24 \mathrm{H}), 0.72-0.62$ $(\mathrm{m}, 16 \mathrm{H}) ;{ }^{13} \mathrm{C} \mathrm{NMR}\left(\mathrm{CDCl}_{3}, 100 \mathrm{MHz}\right) \delta 176.70,65.84$, 39.58, 33.74, 31.77, 26.92, 22.53, 22.44, 17.12, 14.03, 8.7; ${ }^{29} \mathrm{Si}$ NMR $\left(79.5 \mathrm{MHz}, \mathrm{CDCl}_{3}\right) \delta-69.4$. FTIR (neat): $v_{\max }$ $2931,1725,1463,1272,1202,1121,750 \mathrm{~cm}^{-1}$.

Octakis(3-(hexadecanoyloxy)propyl)octasilsesquioxane (2F): ${ }^{1} \mathrm{H} \mathrm{NMR}\left(400 \mathrm{MHz}, \mathrm{CDCl}_{3}\right) \delta$ 4.06-3.95 (m, $\left.16 \mathrm{H}\right)$, 2.33-2.20 (m, $16 \mathrm{H}), 1.75-1.51(\mathrm{~m}, 32 \mathrm{H}), 1.36-1.19$ (m, 192 $\mathrm{H}), 0.88(\mathrm{t}, J=6.56 \mathrm{~Hz}, 24 \mathrm{H}), 0.69-0.56(\mathrm{~m}, 16 \mathrm{H}) ;{ }^{13} \mathrm{C}$ NMR $\left(\mathrm{CDCl}_{3}, 100 \mathrm{MHz}\right) \delta 173.68,65.93,34.31,34.16$, 31.97, 29.75, 29.42, 25.07, 22.70, 22.40, 14.08, 8.63; FTIR (KBr): $v_{\max } 2914,1736,1463,1394,1289,1196,1227,721$ $\mathrm{cm}^{-1}$.

Octaanion Solution, $\left[\mathrm{Me}_{4} \mathbf{N}^{+}\right]_{8}\left[\mathrm{SiO}_{2.5}{ }^{-}\right]_{\mathbf{8}}(\mathbf{8}):^{25}$ To a 250 $\mathrm{mL}$ round bottom flask with a magnetic stir bar, were added 
tetramethylammonium hydroxide $(54.9 \mathrm{~mL}, 0.52 \mathrm{~mol}, 25$ wt \% in $\mathrm{MeOH})$, methanol $(26.66 \mathrm{~mL}, 0.66 \mathrm{~mol})$, and distilled water $(20.1 \mathrm{~mL}, 1.1 \mathrm{~mol})$. The flask was cooled in an ice bath, and the system was maintained under nitrogen. To this mixture, it was then added tetraethoxysilane $(29.36 \mathrm{~mL}$, $0.66 \mathrm{~mol}$ ) via an addition funnel. The solution turned cloudy and allowed to stir at room temperature overnight to obtain a clear solution to obtain tetramethylammonium octaanion $\left[\mathrm{Me}_{4} \mathrm{~N}^{+}\right]_{8}\left[\mathrm{SiO}_{2.5}\right]_{8}$ quantitatively. This solution was characterized by ${ }^{29} \mathrm{Si}$ NMR to realize a peak at $\delta-99.4 \mathrm{ppm}$.

Octa[hydridodimethylsiloxy] octasilsesquioxane, $\left[\mathbf{H M e}_{2} \mathbf{S i O S i O}_{1.5}\right]_{\mathbf{8}}$ or $\mathbf{Q}_{\mathbf{8}} \mathbf{M}_{\mathbf{8}}{ }^{\mathbf{H}} \mathbf{( 9 ) :}^{25}$ To a $250 \mathrm{~mL}$ round bottom flask with a magnetic stirrer, were added hexane $(100 \mathrm{~mL})$ and dimethylchlorosilane $(14.3 \mathrm{~mL}, 0.33 \mathrm{~mol})$ under nitrogen. The flask was placed in an ice bath and 40 $\mathrm{mL}$ of octaanion solution was then added via an addition funnel for an hour. The final mixture was stirred continuously for another $4 \mathrm{~h}$. The two layers were separated with a separator funnel. The hexane layer was dried over anhydrous sodium sulfate, gravity filtered and the solvent removed by rotary evaporation to yield a white powder. This powder was rinsed with methanol and collected by vacuum filtration. The product was dried in a vacuum at $70{ }^{\circ} \mathrm{C}$ for $5 \mathrm{~h}$ to obtain $3.5 \mathrm{~g}$ white powder in $70 \%$ yield. ${ }^{1} \mathrm{H}$ NMR (400 $\mathrm{MHz}$, $\left.\mathrm{CDCl}_{3}\right) \delta 4.73(\mathrm{~m}, 8 \mathrm{H}) 0.25(\mathrm{~d}, 48 \mathrm{H}) .{ }^{13} \mathrm{C} \mathrm{NMR}\left(\mathrm{CDCl}_{3}, 100\right.$ $\mathrm{MHz}) \delta 0.06 .{ }^{29} \mathrm{Si}\left(\mathrm{CDCl}_{3}, 79.5 \mathrm{MHz}\right) \delta-2.9,-108.7$. FTIR $(\mathrm{KBr}): v_{\max } 2966,2144,1258,1096 \mathrm{~cm}^{-1}$.

Octakis(3-hydroxypropyldimethylsiloxy)octasilsesquioxane (OHPS, 10): ${ }^{26} \mathrm{Q}_{8} \mathrm{M}_{8}{ }^{\mathrm{H}}(5.00 \mathrm{~g}, 4.91 \mathrm{mmol})$ was taken in a $100 \mathrm{~mL}$ round bottom flask equipped with a reflux condenser and a magnetic stir bar. It was dissolved in toluene $(25 \mathrm{~mL})$ and was added allyl alcohol $(3.34 \mathrm{~mL}, 49.1$ mmol). The reaction flask was evacuated and refilled with $\mathrm{N}_{2}$ three times. Pt(dvs) (2 mM solution, $\left.1.2 \mathrm{~mL}\right)$ was added via a syringe. The reaction began to reflux immediately due to the exothermic hydrosilylation and was complete in 30 min. After the formation of two layers, triphenylphosphine $(10 \mathrm{mg}$ ) was added to deactivate the $\mathrm{Pt}(\mathrm{dvs})$ catalyst, and the mixture was stirred for $1 \mathrm{~h}$. The bottom layer containing the product, OHPS, was separated and the residual allyl alcohol and toluene were removed under vacuum. mp $84{ }^{\circ} \mathrm{C} .{ }^{1} \mathrm{H}$ NMR (400 MHz, $\left.\mathrm{CDCl}_{3}\right) \delta 3.76(\mathrm{~s}, 8 \mathrm{H}, \mathrm{OH}), 3.54(\mathrm{t}, 16 \mathrm{H})$, 1.59 (p, 16H), $0.58(\mathrm{t}, 16 \mathrm{H}), 0.13(\mathrm{~s}, 48 \mathrm{H}) .{ }^{13} \mathrm{C}$ NMR $(100$ $\left.\mathrm{MHz}, \mathrm{CDCl}_{3}\right) \delta 65.2,26.3,13.6,-0.2 .{ }^{29} \mathrm{Si} \mathrm{NMR}(79.5$ $\left.\mathrm{MHz}, \mathrm{CDCl}_{3}\right) \delta 13.9,-108.4$. FTIR (KBr): $v_{\max } 3339,2959$, $2931,2875,1258,1173,1082 \mathrm{~cm}^{-1}$.

General Procedure for Type-II POSS-Esters. To a mixture of $\mathrm{Et}_{3} \mathrm{~N}$ (2.23 mmol) and octakis(3-hydroxypropyldimethylsiloxy)octasilsesquioxane $(300 \mathrm{mg}, 0.20 \mathrm{mmol})$ in $\mathrm{CH}_{2} \mathrm{Cl}_{2}(10 \mathrm{~mL})$ was added acid chloride $(1.60 \mathrm{mmol})$ at 0 ${ }^{\circ} \mathrm{C}$ under $\mathrm{N}_{2}$. The reaction mixture was stirred for $10 \mathrm{~h}$ at room temperature and then poured into $\mathrm{H}_{2} \mathrm{O}(10 \mathrm{~mL})$. The organic layer was separated, washed with $1 \mathrm{M} \mathrm{HCl}(10 \mathrm{~mL})$, aq. $\mathrm{NaCl}(10 \mathrm{~mL})$, dried over $\mathrm{Na}_{2} \mathrm{SO}_{4}$ and filtered. Evaporation of the organic solvent yielded Type-II POSS-esters.

Octakis[(3-(pentanoyloxy)propyl)dimethylsiloxy]octa silsesquioxane (3A): ${ }^{1} \mathrm{H}$ NMR $\left(400 \mathrm{MHz}, \mathrm{CDCl}_{3}\right) \delta 4.02(\mathrm{t}$,
$J=7.07,16 \mathrm{H}), 2.29(\mathrm{t}, J=7.32,16 \mathrm{H}), 1.70-1.55(\mathrm{~m}, 32 \mathrm{H})$, 1.44-1.29 (m, $16 \mathrm{H}), 0.96-0.88(\mathrm{~m}, 24 \mathrm{H}), 0.64-0.57(\mathrm{~m}, 16$ $\mathrm{H}), 0.16(\mathrm{~s}, 48 \mathrm{H}) ;{ }^{13} \mathrm{C} \mathrm{NMR}\left(\mathrm{CDCl}_{3}, 100 \mathrm{MHz}\right) \delta 174.21$, $66.92,34.38,27.47,26.67,22.80,22.71,14.08,0.41 ;{ }^{29} \mathrm{Si}$ NMR (79.5 MHz, $\left.\mathrm{CDCl}_{3}\right) \delta 14.1,-107.6$. FTIR (neat): $v_{\max }$ 2960, 1736, 1463, 1249, 1173, 1092, $750 \mathrm{~cm}^{-1}$.

Octakis [(3-(hexanoyloxy)propyl)dimethylsiloxy]octa silsesquioxane (3B): ${ }^{1} \mathrm{H} \mathrm{NMR}\left(400 \mathrm{MHz}, \mathrm{CDCl}_{3}\right) \delta 4.02(\mathrm{t}$, $J=7.07,16 \mathrm{H}), 2.28(\mathrm{t}, J=7.57,16 \mathrm{H}), 1.74-1.57(\mathrm{~m}, 32 \mathrm{H})$, 1.39-1.26 (m, $32 \mathrm{H}), 0.94-0.85$ (m, $24 \mathrm{H}), 0.63-0.57$ (m, 16 $\mathrm{H}), 0.15(\mathrm{~s}, 48 \mathrm{H}) ;{ }^{13} \mathrm{C} \mathrm{NMR}\left(\mathrm{CDCl}_{3}, 100 \mathrm{MHz}\right) \delta 174.10$, 66.80, 34.19, 31.94, 25.05, 22.80, 22.72, 14.29, 14.11, 0.40; ${ }^{29} \mathrm{Si} \mathrm{NMR}\left(79.5 \mathrm{MHz}, \mathrm{CDCl}_{3}\right) \delta 14.1,-107.6$. FTIR (neat): $v_{\max } 2960,1736,1463,1260,1173,1092,842 \mathrm{~cm}^{-1}$.

Octakis[(3-cyclohexanecarbonyloxy)propyl)dimethyl siloxy]octasilsesquioxane (3C): $\left.{ }^{1} \mathrm{H} \mathrm{NMR} \mathrm{(400} \mathrm{MHz,} \mathrm{CDCl}_{3}\right)$ $\delta 4.02(\mathrm{t}, J=7.07,16 \mathrm{H}), 2.33-2.23(\mathrm{~m}, 8 \mathrm{H}), 1.95-1.15(\mathrm{~m}$, $96 \mathrm{H}), 0.64-0.54(\mathrm{~m}, 16 \mathrm{H}), 0.15(\mathrm{~s}, 48 \mathrm{H}) ;{ }^{13} \mathrm{C} \mathrm{NMR}\left(\mathrm{CDCl}_{3}\right.$, $100 \mathrm{MHz}) \delta 176.31,66.70,43.55,29.48,26.23,25.90$, 22.76, 14.01, 0.37; ${ }^{29} \mathrm{Si} \mathrm{NMR}\left(79.5 \mathrm{MHz}, \mathrm{CDCl}_{3}\right) \delta 14.4$, -107.9 . FTIR (neat): $v_{\max } 2954,1736,1458,1260,1173$, $1092,750 \mathrm{~cm}^{-1}$.

Octakis[(3-(octanoyloxy)propyl)dimethylsiloxy]octa silsesquioxane (3D): ${ }^{1} \mathrm{H} \mathrm{NMR}\left(400 \mathrm{MHz}, \mathrm{CDCl}_{3}\right) \delta 4.02(\mathrm{t}$, $J=7.07,16 \mathrm{H}), 2.29(\mathrm{t}, J=7.32,16 \mathrm{H}), 1.73-1.55(\mathrm{~m}, 32 \mathrm{H})$, $1.36-1.20(\mathrm{~m}, 64 \mathrm{H}), 0.88(\mathrm{t}, J=6.82,24 \mathrm{H}), 0.66-0.55(\mathrm{~m}$, $16 \mathrm{H}), 0.15(\mathrm{~s}, 48 \mathrm{H}) ;{ }^{13} \mathrm{C} \mathrm{NMR}\left(\mathrm{CDCl}_{3}, 100 \mathrm{MHz}\right) \delta 173.82$, $68.03,33.90,31.75,28.04,25.17,22.76,20.21,14.98$, $14.18,0.45 .{ }^{29} \mathrm{Si}$ NMR $\left(79.5 \mathrm{MHz}, \mathrm{CDCl}_{3}\right) \delta 14.1,-107.6$. FTIR (neat): $v_{\max } 2931,1742,1463,1260,1168,1092,744$ $\mathrm{cm}^{-1}$.

\section{Results and Discussion}

Synthesis of POSS-Type-I esters has been presented in Scheme 1. Octakis(3-chloropropyl)octasilsesquioxane (ClPOSS, 5) was synthesized from commercially available 3chloropropyltrimethoxysilane (CLS, 4) which upon condensation under acidic conditions using concentrated $\mathrm{HCl}$ and catalytic amount of $\mathrm{PtCl}_{4}$ in dry methanol. The POSS-Cl dried under vacuum for $24 \mathrm{~h}$ at $100{ }^{\circ} \mathrm{C}$ to obtain crystalline precipitate in moderate yields. ${ }^{23,24}$ This was then treated with various potassium salts of carboxylic acids in presence of catalytic amount potassium iodide in dry DMF at $100{ }^{\circ} \mathrm{C}$

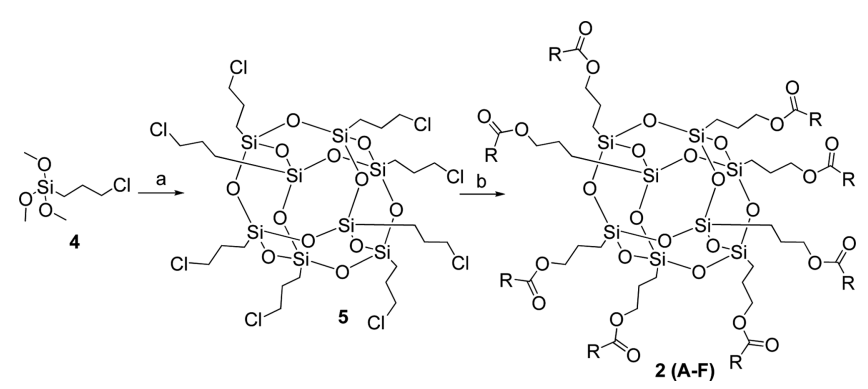

Scheme 1. Preparation of POSS type-I esters 2 (A-F). Reagents and conditions: (a) $\mathrm{HCl}, \mathrm{MeOH}, 25^{\circ} \mathrm{C}$; (b) $\mathrm{RCOO}^{-} \mathrm{K}^{+}$(6), Cat. $\mathrm{KI}$ (5 mol \%), DMF, $100{ }^{\circ} \mathrm{C}$. 
Table 1. Preparation of Type-I POSS-Esters from 5

\begin{tabular}{|c|c|c|c|c|}
\hline Entry & $\begin{array}{c}\text { Potassium } \\
\text { carboxylate (6) }\end{array}$ & Time & Product $^{a}$ & Yield $(\%)^{b}$ \\
\hline 1 & & $48 \mathrm{~h}$ & $2 A$ & 86 \\
\hline 2 & & $48 \mathrm{~h}$ & 2B & 92 \\
\hline 3 & & $48 \mathrm{~h}$ & $2 \mathrm{C}$ & 88 \\
\hline 4 & & $48 \mathrm{~h}$ & 2D & 92 \\
\hline 5 & & $48 \mathrm{~h}$ & $2 E$ & 87 \\
\hline 6 & & $48 \mathrm{~h}$ & $2 F$ & 87 \\
\hline
\end{tabular}

${ }^{a}$ All products were characterized by ${ }^{1} \mathrm{H},{ }^{13} \mathrm{C}$-NMR and FT-IR. ${ }^{b}$ Isolated yields.

gave the type-I POSS esters $\mathbf{2}$ as a odorless, clear viscous oils (Scheme 1) except $\mathbf{2 F}$ which is a light yellow solid, in good yields (Table 1).

To prepare type-II POSS esters, octakis(3-hydroxypropyldimethylsiloxy) octasilsesquioxane (OHPS, 10) was used in esterification with various carboxylic acids (Scheme 2). In this purpose, OHPS was prepared from commercially available tetraethoxysilane (TEOS, 7) in three steps. The TEOS, upon reaction with tetramethylammoniumhydroxide and distilled water in methanol stirred at room temperature for 1 day gave octaanion solution of $\left[\mathrm{Me}_{4} \mathrm{~N}^{+}\right]_{8}\left[\mathrm{SiO}_{2.5}\right]_{8}(\mathbf{8})$ in quantitative yield using Hasegawa's procedure. ${ }^{25}$ The solu-

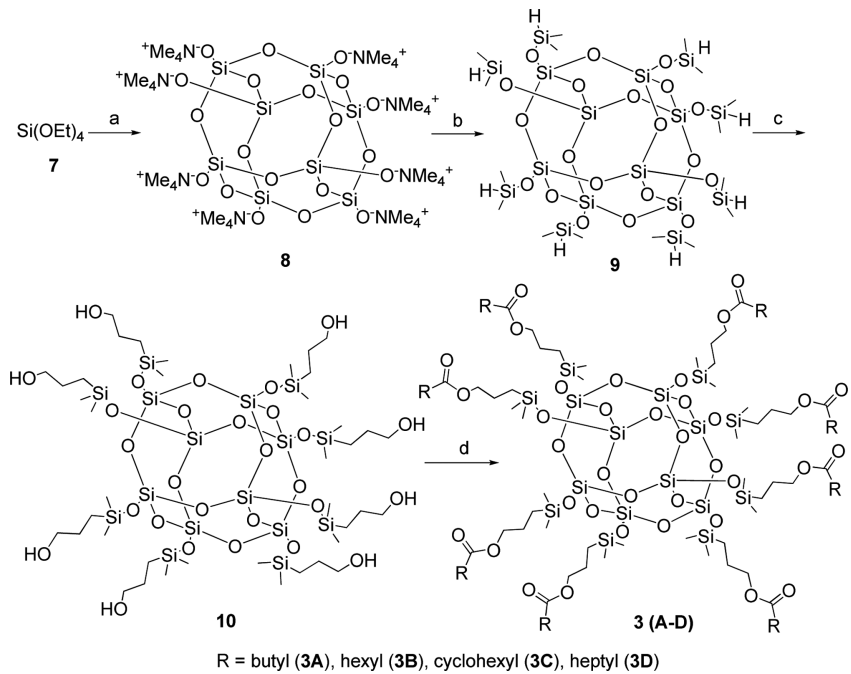

Scheme 2. Preparation of POSS type-II esters. Reagents and conditions: (a) $\mathrm{N}\left(\mathrm{CH}_{3}\right)_{4} \mathrm{OH}, \mathrm{MeOH}, \mathrm{H}_{2} \mathrm{O}$. (b) $\mathrm{HSiMe}_{2} \mathrm{Cl}$, hexane, $0{ }^{\circ} \mathrm{C}$-rt, $4 \mathrm{~h}, 70 \%$; (c) allyl alcohol, $\mathrm{Pt}(\mathrm{dvs})$, dry toluene, $1 \mathrm{~h}, 60 \%$; (d) $\mathrm{RCOCl}(11)$, triethyl amine, $\mathrm{CH}_{2} \mathrm{Cl}_{2}$.
Table 2. Preparation of Type-II POSS-Esters from $\mathbf{1 0}$

\begin{tabular}{|c|c|c|c|c|}
\hline Entry & Acid chloride (11) & Time & Product $^{a}$ & Yield $(\%)^{b}$ \\
\hline 1 & & $10 \mathrm{~h}$ & $\mathbf{3 A}$ & 84 \\
\hline 2 & & $10 \mathrm{~h}$ & 3B & 82 \\
\hline 3 & & $10 \mathrm{~h}$ & $3 C$ & 81 \\
\hline 4 & & $10 \mathrm{~h}$ & 3D & 72 \\
\hline
\end{tabular}

${ }^{a}$ All products were characterized by ${ }^{1} \mathrm{H},{ }^{13} \mathrm{C}$-NMR and FT-IR. ${ }^{b}$ Isolated yields.

tion of octaanion 8 was treated with dimethylchlorosilane in hexane to give octa[hydridodimethylsiloxy] octasilsesquioxane $\left(\left[\mathrm{HMe}_{2} \mathrm{SiOSiO}_{1.5}\right]_{8},(9)\right.$ as white powder after rinsed with methanol. Octakis(3-hydroxypropyldimethylsiloxy)octa silsesquioxane (OHPS, 10) was prepared by direct hydrosilylation of allyl alcohol with 9 using platinum-divinyltetramethyldisiloxane $[\mathrm{Pt}(\mathrm{dvs})]$ as catalyst. ${ }^{26}$ Then, OHPS was subjected to esterification with various acid chlorides in presence of $\mathrm{Et}_{3} \mathrm{~N}$ as base in methylene chloride to give POSS type-II esters 3 (A-D) in good yields (Scheme 2).

After successful synthesis of two types of POSS esters, we have studied Thermo Gravimetric Analysis (TGA) for three compounds to investigate thermal characteristics of these classes. The thermal decomposition of POSS-esters was conducted by TGA under nitrogen atmosphere for 25-1000 ${ }^{\circ} \mathrm{C}$. The graphical representation of weight loss to temperature has been shown in Figure 2. The TGA spectra show that all POSS-Esters are stable below $350^{\circ} \mathrm{C}$ and decompose after $450{ }^{\circ} \mathrm{C}$. Further, it can be seen that type-II esters are more thermally stable when compared to type-I. However, these ester compounds (type-I \& II) possess promising environmentally friendly oil characteristics that come from

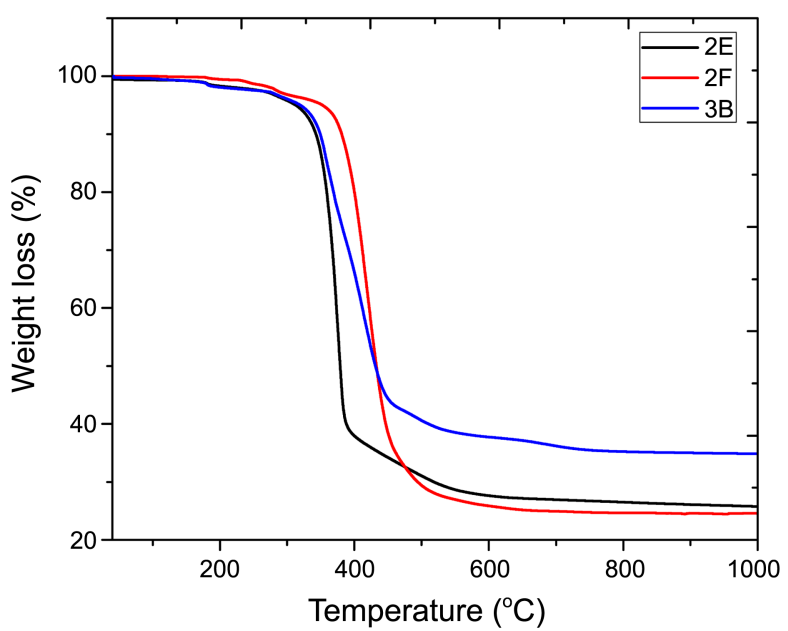

Figure 2. TGA spectra of compounds $\mathbf{2 E}, \mathbf{2 F}$ and $\mathbf{3 B}$. 
POSS skeleton even at elevated temperatures, so that they can be used in transformer insulation system and expected to serve as additives to some insulation oils.

\section{Conclusions}

Novel Hybrid polyoctahedral silsesquioxane (POSS)-based type-I and type-II esters have been synthesized as alternatives to mineral and vegetable oils. These substances are prepared from Cl-POSS, OHPS and respective carboxylic acids. These POSS esters are environmentally friendly. All the compounds were completely characterized by ${ }^{1} \mathrm{H} \&{ }^{13} \mathrm{C}$ NMR and FT-IR spectroscopic analysis. Thermal decompositions of three new compounds were discussed using TGA as a preliminary investigation on their thermal properties.

Acknowledgments. This work was supported by the grants from Ministry of Environment (KME, 412-111-008), National Research Foundation (NRF-2009-0094063) and Ministry of Knowledge Economy (MKE, R0000495), South Korea.

\section{References}

1. Booth, C.; McDonald, J. R. Neurocomputing 1998, 23, 97-109.

2. Eisentraeger, A.; Schimidt, M.; Murrenhoff, H.; Dott, W.; Hahn. S. Chemosphere 2000, 41, 1011-1027.

3. Inmaculada, F.; Alfredo, O.; Fernando, D.; Carlos, R.; Severiano, P. Elec. Pow. Sys. Res. 2013, 98, 58-69.

4. Oommen, T. V. IEEE Electrical Insulation Magazine 2002, 18, 611.

5. Lin, S. S. AOCS Publication 1997, 211-231.

6. Pavlov, D. J.; Gospodinova, N. N.; Glavchev, I. K. Ind. Lubr.
Tribol. 2004, 56, 19-22.

7. Kuo, S. W.; Chang, F. C. Prog. Polym. Sci. 2011, 36, 1649-1696.

8. Cordes, D. B.; Lickiss, P. D.; Rataboul, F. Chem. Rev. 2010, 110, 2081-2173

9. Lee, Y. J.; Huang, J. M.; Kuo, S. W.; Lu, J. S; Chang, F. C. Polymer 2005, 46, 173-181.

10. Zhang, Z.; Gu, A.; Liang, G.; Ren, P.; Xie, J.; Wang, X. Polym. Degrad. Stab. 2007, 92, 1986-1993.

11. Tang, Y.; Lewin, M. Polym. Advan. Technol. 2009, 20, 1-15.

12. Zhang, B.; Chen, Y.; Wang, J.; Blau, W. J.; Zhuang, X; He, N. Carbon 2010, 48, 1738-1742.

13. Liu, W. C.; Yu, Y. Y.; Chen, W. C. J. Appl. Polym. Sci. 2004, 91, 2653-2660.

14. Prem Kumar, B.; Vijaykumar, B. V. D.; Raghavan, C. M.; Harshavardhan, S. J.; Yi, S.-S.; Sakthivel, G.; Jiang, J.; Jang, K.; Shin, D.-S. J. Mater. Sci. 2013, 48, 7533-7539.

15. Leland, J. V.; Brent, P.; Shawn, H.; Blanski, R. In: International SAMPE Symposium and Exhibition Proceedings 2002, $47 \mathrm{II}$; 1503-1507.

16. Liu, Y.; Zheng, S.; Nie, K. Polymer 2005, 46, 12016-12025.

17. Dodiuk, H.; Kening, S.; Blinsky, I.; Dotan, A.; Buchman, A. Int J. Adhes. Adhes. 2005, 25, 211-218.

18. Zou, Q.-C.; Yan, Q.-J.; Song, G.-W.; Zhang, S.-L.; Wu, L.-M. Biosens. Bioelectron. 2007, 22, 1461-1465.

19. Skaria, S.; Schricker, S. R. J. Macromol. Sci., Part A: Pure and Appl. Chem. 2010, 47, 381-391.

20. Kim, S. K.; Heo, S. J.; Koak, J. H.; Lee, Y. M.; Chung, D. J.; Lee, J. I.; Hong, S. D. J. Oral Rehabil. 2007, 34, 389-395.

21. Punshon, G.; Vara, D. S.; Sales, K. M.; Kidane, A. G.; Salacinski, H. J.; Seifalian, A. M. Biomaterials 2005, 26, 6271-6279.

22. Seifalian, A.; Salacinski, H.; Srai, K.; Ramesh, B.; Darbyshire, A.; Hancock, S. WO 2005070988, August 2005.

23. Dittmar, U.; Hendon, B. J.; Floerke, U.; Marsmann, H. C. J. Organomet. Chem. 1995, 489, 185-194.

24. Gultek, A. Turk J. Chem. 2010, 34, 437-445.

25. Hasegawa, I.; Motojima, S. J. Organomet. Chem. 1992, 441, 373380 .

26. Zhang, C.; Laine, R. M. J. Am. Chem. Soc. 2000, 122, 6979-6988. 